Journal homepage http://revistas.unitru.edu.pe/index.php/SSMM

\begin{tabular}{|c|c|c|}
\hline & $\begin{array}{c}\text { SELECCIONES MATEMÁTICAS } \\
\text { Universidad Nacional de Trujillo } \\
\text { ISSN: } 2411-1783 \text { (Online) } \\
\text { Vol. 05(01): } 85-101(2018)\end{array}$ & 回招回 \\
\hline
\end{tabular}

\title{
Análisis de la Solución Numérica en la Ecuación de Difusión no Estacionaria Unidimensional usando Esquemas de Diferencias Finitas Miméticas y Crank Nicolson
}

\section{Analysis of the Numerical Solution in the One Dimensional Non-Stationary Diffusion Equation using Schemes of Finite Mimetic Differences and Crank Nicolson}

\author{
${\text { Mardo Gonzales Herrera* }{ }^{*} \text { and Saulo Murillo Cornejo }}^{\dagger}$
}

Received, Feb. 14, 2018

Accepted, Jun. 10, 2018

DOI: http://dx.doi.org/10.17268/sel.mat.2018.01.10

\section{Resumen}

Se propone la solución numérica de la ecuación de difusión no estática unidimensional, desarrollando un algoritmo en software Matlab versión 7.0, para lo cual se combina el esquema de diferencias finitas miméticas en la aproximación de los operadores diferenciales del continuo (gradiente y divergencia) para la variable espacial, sobre una malla uniforme, cuyos operadores diferenciales discretos presentan una aproximación de segundo orden y el enfoque en diferencias finitas tipo Crank Nicolson para obtener aproximaciones en la variable temporal.

Este algoritmo propuesto para los enfoques miméticos y Crank Nicolson presentan mejor aproximación que el esquema en diferencias finitas tipo Crank Nicolson.

Además se calcula el error de aproximación generado entre la solución numérica y la solución analítica usando la norma del máximo para la ecuación de difusión no estacionaria con condiciones de frontera tipo Robin.

Palabras clave. Algoritmo en matlab, Operadores miméticos, Ecuación de difusión, Método de diferencias finitas iméticas, Esquema Crank Nicolson.

\begin{abstract}
The numerical solution of the one-dimensional non-static diffusion equation is proposed, developing an algorithm in software Matlab version 7.0, for which the mimetic finite difference scheme is combined in the approximation of the differential operators of the continuum (gradient and divergence) for the spatial variable, on a uniform grid, whose discrete differential operators have a second order approximation and the finite difference approach type Crank Nicolson for approximations in the temporary variable.

This proposed algorithm for the mimetic and Crank Nicolson approaches has a better approximation than the finite-difference Crank Nicolson-type scheme.

In addition, the approximation error generated between the numerical solution and the analytical solution is calculated using the maximum standard for the non-stationary diffusion equation with Robin type boundary conditions.
\end{abstract}

Keywords. Algorithm in matlab, Mimetic operators, Diffusion equation, Mimetic finite difference method, Crank

\footnotetext{
*Universidad Nacional Pedro Ruiz Gallo, Ca. Juan XXII. s/n, Lambayeque. Perú (mardo . unprg@ gmai 1 . com) .

${ }^{\dagger}$ Escuela de posgrado UNT ( sauloedison.mc@gmail. com

This work is licensed under the Creative Commons Attribution-NoComercial-ShareAlike 4.0.
} 
Nicolson scheme

1. Introducción. En los últimos quince años una nueva versión del método en diferencias finitas, denominado mimético, ha venido desarrollándose con la finalidad de obtener mejores resultados para la simulación de diversos fenómenos.

Se resolvieron muchos problemas haciendo uso del método en diferencias finitas miméticas, donde se construyeron operadores diferenciales miméticos a nivel unidimensional, sin embargo, afirman que la creación de aproximaciones de segundo orden fuera de la frontera es simple, pero que tener un comportamiento adecuado alrededor de la frontera es difícil, incluso en una rejilla unidimensional uniforme [2].

Esta nueva versión, goza de una ventaja al mantener los mismos ordenes de exactitud de los operadores discretos tanto en los nodos internos como en la frontera de la malla y además satisfacen versiones discretas de una ley de conservación global (Teorema de divergencia) convirtiéndolos así en métodos conservativos [3].

Una forma discreta de la ley de conservación se necesita ser construida para satisfacer la conservación local en cada intervalo de la célula, de tal manera que se cumpla la ley de conservación global para todo el intervalo investigado [2].

En tal sentido el esquema de diferencias finitas miméticas en sus distintas aplicaciones, siempre preserva propiedades de la ley de conservación, simetría y positividad de las soluciones. Debido a estas propiedades se presentó una alternativa que ofrecen estos esquemas miméticos.

Se creó una aplicación implementada por el método de diferencias finitas miméticas el cual se utilizó para simular escenarios de almacenamiento de carbono en medios geológicos, debido a las características de los operadores discretos en preservar propiedades de sus pares continuos, la solución a ecuaciones de conservación, como las que modelan los perfiles de concentración de soluto en el almacenamiento de carbono son beneficiados usando este enfoque mimético [8] y [7].

Como es habitual en la tecnología numérica mimética, el método satisface las condiciones locales de consistencia y estabilidad los cuales determinan la precisión y el buen estado de la aproximación resultante, pues esta nueva familia de métodos miméticos demuestra una buena convergencia y estimaciones optima de error [9]. Luego se proporciona una aplicación del método de diferencias finitas miméticas para el flujo en las fracturas de datos micro sísmico.

La idea principal es presentar la descripción e investigación de un nuevo algoritmo usando un nuevo enfoque del método mimético [1] para determinar la solución aproximada a la ecuación del calor no estacionaria unidimensional.

\subsection{Ecuación del Modelo Continuo.}

La forma general de la ecuación no estática del calor unidimensional es dada por:

$$
\frac{\partial u}{\partial t}(x, t)=\nabla \cdot(K \nabla u(x, t))+F(x, t)
$$

donde $F \in C([0,1] \times[0, T])$ es el término fuente conocido, $u$ es la función incógnita de temperatura y $K$ es el término coeficiente de difusividad.

De tal manera que el problema estará bien puesto si garantizamos existencia y unicidad de la solución, y en ese sentido aplicamos la condición mixta de Robín.

$$
\alpha(x) u(x, t)+\frac{\partial u}{\partial n}(x, t)=f(x, t) \quad, \quad f(x, t) \in C\left(S_{T}\right)
$$

con la condición inicial

$$
u(x, 0)=f(x) \quad, \quad f(x) \in C([0,1])
$$

\section{Definimos los espacios de funciones.}

$C([0,1])=\{f:[0,1] \rightarrow R /$ f es continua $\}$

$C_{1}^{2}([0,1] \times[0, T])=\left\{u:[0,1] \times\left[0, \infty>\rightarrow R / u, D_{x} u, D_{x}^{2} u, u_{t} \in C([0,1] \times[0, \infty>)\}\right.\right.$

$S_{T}=\left\{(x, t) \in R^{2} / x \in \partial \Omega, 0 \leq t<T\right\}$

$C\left(S_{T}\right)=\left\{f: S_{T} \rightarrow R /\right.$ f es continua $\}$ 


\subsection{Discretización Mimética de Operadores Diferenciales.}

Usaremos una analogía discreta del teorema de la divergencia para determinar el gradiente discreto.

El teorema de la divergencia dice que:

$$
\int_{\Omega} \nabla \cdot \vec{v} f d V+\int_{\Omega} \vec{v} \nabla f d V=\int_{\partial \Omega} f \vec{v} \vec{n} d S
$$

donde $\Omega$ es una región, $\partial \Omega$ es la frontera de la región, $\vec{n}$ es la normal exterior a la frontera, $f$ es una función escalar bien definida sobre la cerradura de la región $\Omega$ y $v$ es un campo vectorial suave definido sobre la cerradura de la región $\Omega$.

Si $f, g$ son campos escalares y si $\vec{v}, \vec{w}$ son campos vectoriales, los productos internos adecuados del continuo para escalares y vectores son:

$$
\langle f, g\rangle=\int_{\Omega} f g d V \quad\langle\vec{v}, \vec{w}\rangle=\int_{\Omega} \vec{v} \vec{w} d V
$$

entonces (1.4) puede escribirse:

$$
\langle\nabla \cdot \vec{v}, f\rangle+\langle\vec{v}, \nabla f\rangle=\int_{\partial \Omega} f \vec{v} \vec{n} d S
$$

El teorema de la divergencia en una dimensión, se convierte en una integración por partes.

$$
\int_{0}^{1} \frac{d v}{d x} f d x+\int_{0}^{1} v \frac{d f}{d x} d x=v(1) f(1)-v(0) f(0)
$$

Una forma discreta de la ley de conservación necesita ser construida para satisfacer la conservación local en cada intervalo de la célula, de tal manera que se cumpla la ley de conservación global para todo el intervalo investigado.

1.2.1. Definición. Sea $\vec{v}: R \rightarrow R^{N+1}$ una función vectorial discreta, definida sobre los nodos de la malla unidimensional, tal que $v(t)=\left(v_{0}(t), v_{1}(t), \cdots, v_{N}(t)\right) \forall t \in R, D_{v} \in R^{N}$ representa la aproximación en los centros de las celdas $\nabla \vec{v}$, se define la divergencia en los centros de la célula como:

$$
\begin{aligned}
D_{v} \subset R^{N+1} & \rightarrow R^{N} \\
\left(D_{v}\right)_{i+\frac{1}{2}} & =\frac{\left(v_{i+1}-v_{i}\right)}{h} \quad \text { para } i=0,1,2, \cdots, n-1
\end{aligned}
$$

las aproximaciones de la divergencia en los centros de las celdas coinciden con el esquema en diferencias central, la cual expresa una matriz $D_{(N) \times(N+1)}$

1.2.2. Definición. Sea $f=\left(f_{0}, f_{1 / 2}, f_{3 / 2}, \cdots, f_{N-1 / 2}, f_{N}\right)^{T} \in R^{N+2}$ una función discreta definida en los centros de la célula y en la frontera del dominio de la malla unidimensional, $G f \in R^{N+1}$ representa las aproximaciones en los nodos $\nabla f$.

Se define el gradiente $G$ en una malla unidimensional como:

$$
G f \subset R^{N+2} \rightarrow R^{N+1} \text { tal que }
$$

el gradiente en la frontera tiene la forma

$$
\begin{aligned}
(G f)_{0} & =\frac{-\frac{8}{3} f_{0}+3 f_{\frac{1}{2}}-\frac{1}{3} f_{\frac{3}{2}}}{h} \\
(G f)_{N} & =\frac{\frac{8}{3} f_{N}-3 f_{N-\frac{1}{2}}+\frac{1}{3} f_{N-\frac{3}{2}}}{h}
\end{aligned}
$$

mientras que en los puntos interiores, la aproximación del gradiente coincide con el esquema en diferencia central, es decir:

$$
(G f)_{i}=\frac{f_{i+\frac{1}{2}}-f_{i-\frac{1}{2}}}{h} \quad \text { para } i=1,2, \cdots n-1,
$$


la cual expresa una matriz $G_{(N+1) \times(N+2)}$

Observación 1. Ahora que se tiene los operadores en forma de matriz vamos a reformular las condiciones miméticas de los operadores discretos gradiente $(G)$ y divergencia $(D)$.

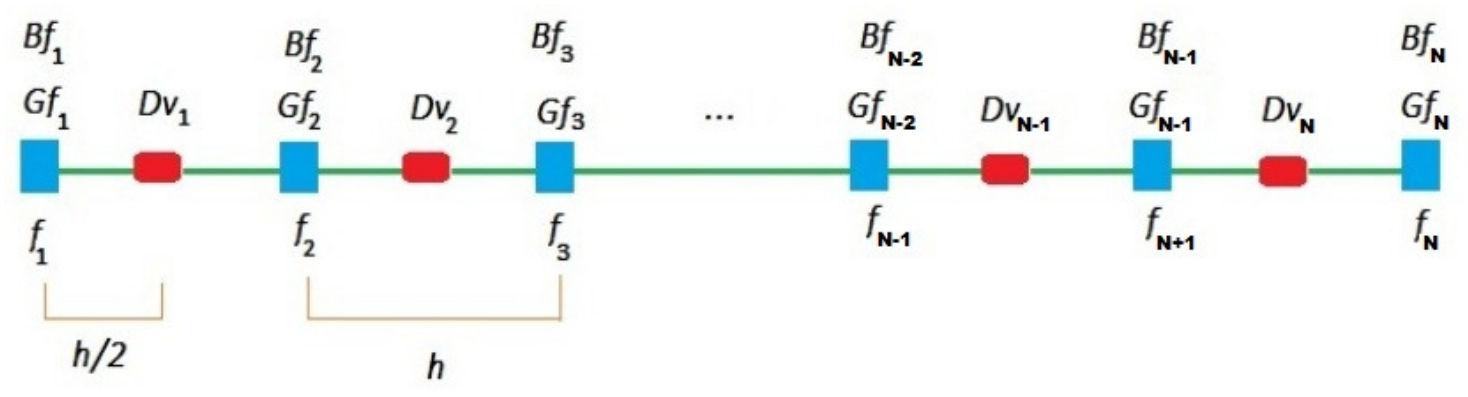

FIGURA 1.1. Malla Escalonada Uniforme Unidimensional

Donde $(D)$ indica la aproximación de diferencias para el operador divergencia tal como se aplica a la función en los centros de la célula y $(G)$ denota la aproximación de diferencias para el operador gradiente tal como se aplica a la función en los nodos de la célula y la frontera.

En la figura (1.1) el espaciamiento de la célula es uniforme con una longitud de $h=\frac{1}{N}$ sobre el intervalo $[0 ; 1]$. El intervalo investigado es dividido igualmente en $n$ sub intervalos, cada nodo tiene una coordenada $x_{i}=(i * h)$ con $0 \leq i \leq N$.

Cada célula tiene un punto central, es decir, $\left[x_{i}, x_{i+1}\right]$ incluye el centro de coordenada $x_{i+1 / 2}$.

La forma discreta del operador gradiente $(G)$ en los bordes internos, así como en la frontera es dado en [2] $\mathrm{y}[3]$

$$
(G u)_{i}=\frac{u_{1+\frac{1}{2}}-u_{1+\frac{1}{2}}}{h} \quad 1 \leq i \leq N
$$

$$
\begin{aligned}
(G u)_{0} & =\frac{-8}{3 h} u_{0}+\frac{3}{h} u_{\frac{1}{2}}-\frac{1}{3 h} u_{\frac{3}{2}} \\
(G u)_{N} & =\frac{8}{3 h} u_{N}-3 u_{N-\frac{1}{2}}+\frac{1}{3 h} u_{N-\frac{3}{2}}
\end{aligned}
$$

La discretización mimética de la divergencia $(D)$ en los centros de los bloques esta dada:

$$
(D v)_{i+\frac{1}{2}}=\frac{v_{i+1}-v_{i}}{h} \quad 0 \leq i \leq N
$$

1.3. Teorema de la divergencia discreta enfoque Castillo-Grone [2] [3]. En general el enfoque Castillo-Grone dado en (1.4) expresan la ley de conservación de la ecuación (1.6) la cual se representa en forma de un producto interno ponderado de funciones escalares y vectoriales discretas sobre rejillas escalonadas, la relación dada es:

$$
\langle\widehat{D} v, f\rangle_{Q}+\langle v, G f\rangle_{P}=\langle B v, f\rangle_{I}
$$

donde $D, G$ y $B$ son las versiones discretas de sus continuos correspondientes: gradiente $(\nabla)$, divergencia $\left(\nabla\right.$.) y operador de frontera $\left(\frac{\partial}{\partial \vec{n}}\right)$. El $\langle$,$\rangle representa un producto interior generalizado con pesos Q, P$ e $I$. Usando la identidad (1.12) se obtiene una relación para el operador de frontera.

$$
B=Q \widehat{D}+G^{t} P
$$

además $[Q],[P]$ y $[I]$ son matrices definidas positivas cuyo orden es $(N+2) \times(N+2),(N+1) \times(N+1)$ y $(N+2) \times(N+2)$ respectivamente, usadas para determinar la forma de $\widehat{D}$ y $G$. 
Los operadores discretos de segundo orden introducidos por Castillo-Grone son dados en [2] , [3] y [5]

$$
\begin{gathered}
G=\frac{1}{h}\left(\begin{array}{ccccccccc}
\frac{-8}{3} & 3 & \frac{-1}{3} & 0 & \cdots & \cdots & \cdots & 0 & 0 \\
0 & -1 & 1 & 0 & \cdots & \cdots & \cdots & 0 & 0 \\
0 & 0 & -1 & 1 & \cdots & \cdots & \cdots & 0 & 0 \\
\vdots & \vdots & \vdots & \vdots & \ddots & \ddots & \cdots & 0 & 0 \\
\vdots & \vdots & \vdots & \vdots & \cdots & -1 & 1 & 0 & 0 \\
\vdots & \vdots & \vdots & \vdots & \cdots & 0 & -1 & 1 & 0 \\
0 & \cdots & \cdots & \cdots & \cdots & 0 & \frac{1}{3} & -3 & \frac{8}{3}
\end{array}\right)_{(N+1) \times(N+2)} \\
\widehat{D}=\left(\begin{array}{ccccccc}
0 & 0 & 0 & \cdots & 0 & 0 \\
-\frac{1}{h} & \frac{1}{h} & 0 & \cdots & 0 & 0 \\
0 & -\frac{1}{h} & \frac{1}{h} & \cdots & 0 & 0 \\
\vdots & \vdots & \ddots & \ddots & \vdots & \vdots \\
0 & 0 & \cdots & -\frac{1}{h} & \frac{1}{h} & 0 \\
0 & 0 & 0 & \cdots & -\frac{1}{h} & \frac{1}{h} \\
0 & 0 & 0 & \cdots & 0 & 0
\end{array}\right)_{(N+2) \times(N+1)}
\end{gathered}
$$

Aparecen dos filas de ceros en $\widehat{D}$ (una en la parte superior y otra en la parte inferior) con esto se busca obtener una matriz cuadrada al componer operadores, el operador de frontera $B$ queda dado explícitamente.

$$
B=\left(\begin{array}{ccccccc}
-1 & 0 & 0 & \cdots & 0 & 0 & 0 \\
\frac{1}{8} & -\frac{1}{8} & 0 & \cdots & 0 & 0 & 0 \\
-\frac{1}{8} & \frac{1}{8} & 0 & \cdots & 0 & 0 & 0 \\
0 & 0 & 0 & \cdots & 0 & 0 & 0 \\
\vdots & \vdots & \vdots & \ddots & \vdots & \vdots & \vdots \\
0 & 0 & 0 & \cdots & 0 & 0 & 0 \\
0 & 0 & 0 & \cdots & 0 & -\frac{1}{8} & \frac{1}{8} \\
0 & 0 & 0 & \cdots & 0 & \frac{1}{8} & -\frac{1}{8} \\
0 & 0 & 0 & \cdots & 0 & 0 & 1
\end{array}\right)_{(N+2) \times(N+2)}
$$

Por lo tanto, los operadores miméticos gradiente $(G)$ y divergencia $(\widehat{D})$ son de segundo orden tanto en el interior del dominio como en la frontera sobre una malla uniforme escalonada unidimensional.

El esquema mimético para la ecuación de difusión (1.1) sujeto a las condiciones de contorno tipo Robín (1.2) queda dado por:

$$
M I \equiv(\widehat{A}+B G+\widehat{D} K G) f=b
$$

donde $\widehat{A}$ es una matriz de orden $(N+2) \times(N+2)$ que tiene entradas distintas de cero en su diagonal, que corresponden a los nodos de la frontera. Los valores asociados a las entradas son no nulas.

Para el caso unidimensional las únicas entradas no nulas son:

$$
\widehat{A}(1,1)=\alpha_{0} \quad \mathbf{y} \quad \widehat{A}(N+2, N+2)=\alpha_{1}
$$

El operador $K$ es un tensor diagonal cuyos valores conocidos son positivos y evaluada en los bordes de la red. A veces el producto $(K G) f$ se llama flujo. El vector $\vec{b}$ tiene la forma:

$$
\vec{b}=\left(\gamma_{0}, F_{\frac{1}{2}}, F_{\frac{3}{2}}, \cdots, F_{n-\frac{1}{2}}, \gamma_{1}\right)^{T}
$$

$f$ representa la aproximación mimética.

El nuevo método mimético dado en [1] para la ecuación del calor unidimensional, se basa especificamente sobre el esquema de Crank Nicolson para la variable temporal y el esquema de diferencias finitas miméticas para la variable espacial. 
2. Esquema Mimético de Crank Nicolson. El esquema de Crank Nicolson propuesto en [1] se obtiene al combinar el esquema en diferencias implícito con el esquema explícito en la malla mimética.

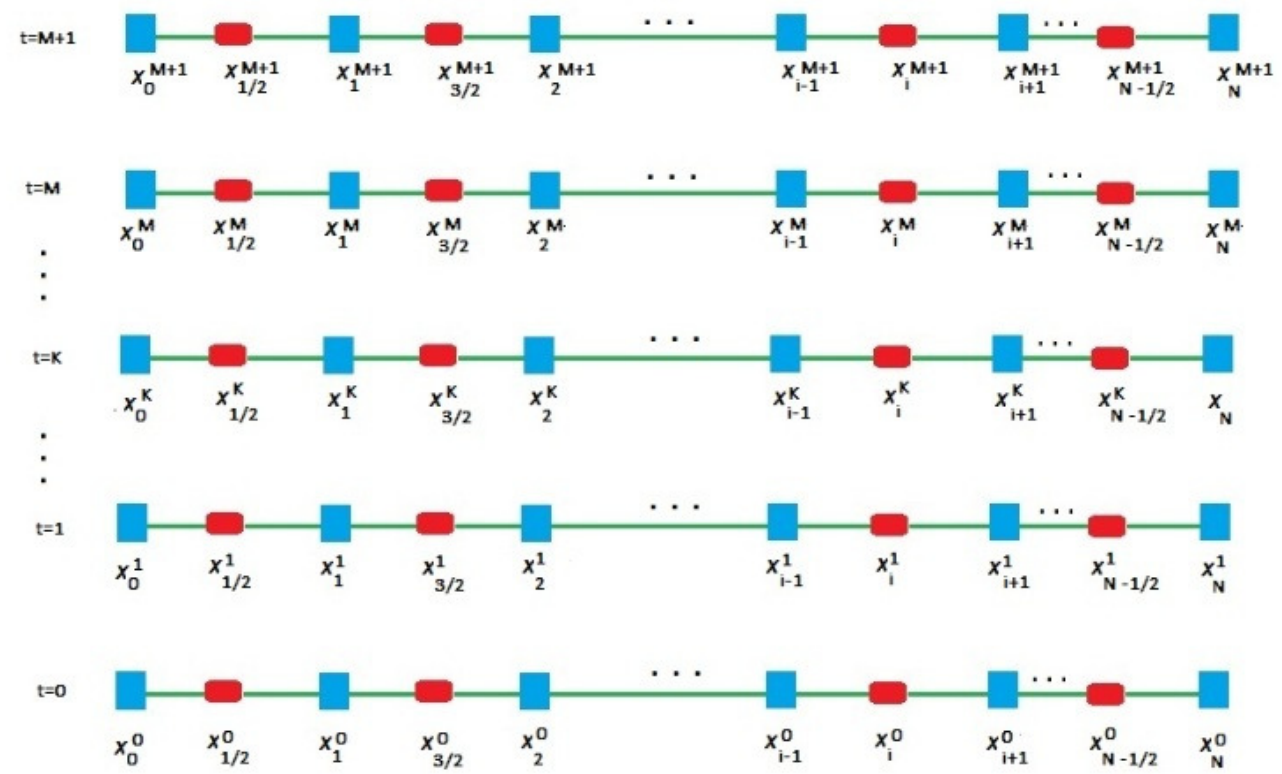

FIGURA 2.1. Molecula Mimética Computacional

El esquema de diferencias Implícito para la variable tiempo es dado :

$$
\frac{\partial u}{\partial t} u(x, t)=2[T]\left(U^{N+\frac{1}{2}}-U^{N}\right)+\vartheta(\Delta t)
$$

donde $[T]=\frac{1}{\Delta t}$.

Luego de la ecuación (1.1), se obtiene:

$$
\begin{aligned}
2[T]\left(U^{N+\frac{1}{2}}-U^{N}\right) & =D\left(K G U^{N}\right)+F^{N} \\
2[T]\left(U^{N+\frac{1}{2}}-U^{N}\right)-[D][K][G] U^{N} & =F^{N}
\end{aligned}
$$

Además incorporando las condiciones de frontera (1.2), en la malla mimética obtenemos:

$$
(\alpha[A]+\beta[B G]) U^{N}=f^{N}
$$

donde $[\mathrm{T}]$ es una matriz diagonal cuyas entradas diferentes de cero son de la forma $\frac{1}{\Delta t}$.

Los vectores $U$ y $F$ son las aproximaciones de la temperatura y la fuente sobre los nodos en la malla mimética.

El esquema explicito tendría la siguiente forma:

$$
\frac{\partial u}{\partial t} u(x, t)=2[T]\left(U^{N+1}-U^{N+\frac{1}{2}}\right)+\vartheta(\Delta t)
$$

donde $[T]=\frac{1}{\Delta t}$.

Luego de la ecuación (1.1), se obtiene:

$$
\begin{aligned}
2[T]\left(U^{N+1}-U^{N+\frac{1}{2}}\right) & =[D][K][G] U^{N+1}+F^{N+1} \\
2[T]\left(U^{N+1}-U^{N+\frac{1}{2}}\right)-[D][K][G] U^{N+1} & =F^{N+1}
\end{aligned}
$$

Además incorporando las condiciones de frontera (1.2), en la malla mimética obtenemos:

$$
(\alpha[A]+\beta[B G]) U^{N+1}=f^{N+1}
$$


Combinando las expresiones (2.2)y(2.3) se tiene:

$$
2[T] U^{N+\frac{1}{2}}-2[T] U^{N}=([D][K][G]-\alpha[A]-\beta[B][G]) U^{N}+F^{N}+f^{N}
$$

Combinando las expresiones (2.5)y (2.6) se tiene:

$$
2[T] U^{N+1}-2[T] U^{N+\frac{1}{2}}=([D][K][G]-\alpha[A]-\beta[B][G]) U^{N+1}+F^{N+1}+f^{N+1}
$$

Sumando las expresiones (2.7) y (2.8) tenemos:

$$
\begin{aligned}
{[T] U^{N+1}-[T] U^{N} } & =\frac{1}{2}([D][K][G]-\alpha[A]-\beta[B][G]) U^{N}+\frac{1}{2}\left(F^{N}+f^{N}\right) \\
& +\frac{1}{2}([D][K][G]-\alpha[A]-\beta[B][G]) U^{N+1}+\frac{1}{2}\left(F^{N+1}+f^{N+1}\right) \\
\left([T]-\frac{1}{2}([D][K][G]-\alpha[A]-\beta[B][G])\right) U^{N+1} & =\left([T]+\frac{1}{2}([D][K][G]-\alpha[A]-\beta[B][G])\right) U^{N} \\
& +\frac{1}{2}\left(F^{N+1}+F^{N}\right)+\frac{1}{2}\left(f^{N+1}+f^{N}\right)
\end{aligned}
$$

De la expresión dada en (2.9), vemos que se obtiene un sistema de $(N+2) \times(N+2)$, es decir que este nuevo esquema presentado por [1] contiene $(N)$ ecuaciones lineales, uno por cada nodo interno y dos ecuaciones para la condición de frontera.

A continuación presentamos un caso particular para $(N=5)$, puntos en el dominio.

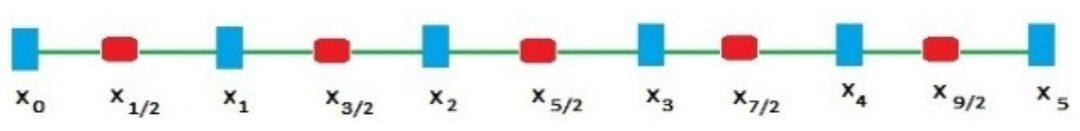

FIGURA 2.2. Discretización del dominio para $(N=5)$

\section{Aproximación en el primer nodo $\left(x_{0}\right)$}

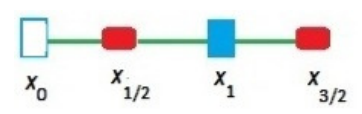

FIGURA 2.3. Aproximación en la frontera izquieda

De la ecuación (2.9) se obtiene la aproximación en la frontera izquierda:

$$
\begin{aligned}
\left(\frac{1}{2} \alpha_{1}+\frac{4}{3 h}\right) U_{0}^{N+1}-\left(\frac{3}{2 h}\right) U_{\frac{1}{2}}^{N+1}+\left(\frac{1}{6 h}\right) U_{\frac{3}{2}}^{N+1} & =\left(-\frac{1}{2} \alpha_{1}-\frac{4}{3 h}\right) U_{0}^{N}+\left(\frac{3}{2 h}\right) U_{\frac{1}{2}}^{N}-\left(\frac{1}{6 h}\right) U_{\frac{3}{2}}^{N} \\
& +\frac{1}{2}\left(f_{0}^{N}+f_{0}^{N+1}\right)
\end{aligned}
$$

la cual representa una discretización de segundo orden incluyendo las condiciones de frontera en el nodo $x_{0}$.

\section{Aproximación en el segundo nodo $x_{\frac{1}{2}}$}

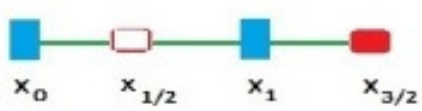


Aplicando la ecuación (2.9) en el primer punto medio, resulta.

$$
\begin{aligned}
\left(-\frac{1}{6 h}-\frac{4}{3 h^{2}}\right) U_{0}^{N+1} & +\left(\frac{1}{k}+\frac{1}{4 h}+\frac{2}{h^{2}}\right) U_{\frac{1}{2}}^{N+1}+\left(-\frac{1}{12 h}-\frac{2}{3 h^{2}}\right) U_{\frac{3}{2}}^{N+1}=\left(\frac{1}{6 h}+\frac{4}{3 h^{2}}\right) U_{0}^{N} \\
& +\left(\frac{1}{k}-\frac{1}{4 h}-\frac{2}{h^{2}}\right) U_{\frac{1}{2}}^{N}+\left(\frac{1}{12 h}+\frac{2}{3 h^{2}}\right) U_{\frac{3}{2}}^{N}+\frac{1}{2}\left(F_{\frac{1}{2}}^{N}+F_{\frac{1}{2}}^{N+1}\right)
\end{aligned}
$$

\section{Aproximación en el tercer nodo $x_{\frac{3}{2}}$}

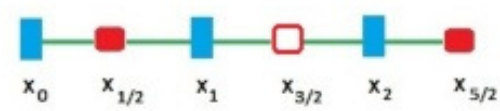

FIGURA 2.5. Aproximación en el segundo punto medio

Aplicando la ecuación (2.9) en el segundo punto medio, resulta.

$$
\begin{aligned}
\left(\frac{1}{6 h}\right) U_{0}^{N+1} & +\left(-\frac{1}{4 h}-\frac{1}{2 h^{2}}\right) U_{\frac{1}{2}}^{N+1}+\left(\frac{1}{k}+\frac{1}{12 h}+\frac{1}{h^{2}}\right) U_{\frac{3}{2}}^{N+1}-\left(\frac{1}{2 h^{2}}\right) U_{\frac{5}{2}}^{N+1}=\left(-\frac{1}{6 h}\right) U_{0}^{N} \\
& +\left(\frac{1}{4 h}+\frac{1}{2 h^{2}}\right) U_{\frac{1}{2}}^{N}+\left(\frac{1}{k}-\frac{1}{12 h}-\frac{1}{h^{2}}\right) U_{\frac{3}{2}}^{N}+\left(\frac{1}{2 h^{2}}\right) U_{\frac{5}{2}}^{N}+\frac{1}{2}\left(F_{\frac{3}{2}}^{N}+F_{\frac{3}{2}}^{N+1}\right)
\end{aligned}
$$

Aquí observamos que la discretización en el punto $\left(x_{\frac{3}{2}}\right)$ presenta cuatro términos en esta ecuación.

\section{Aproximación en el cuarto nodo $x_{\frac{5}{2}}$}

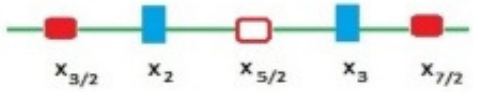

FIGURA 2.6. Aproximación en el tercer punto medio

Aplicando la ecuación (2.9) en el segundo punto medio, resulta.

$$
\begin{aligned}
\left(-\frac{1}{2 h^{2}}\right) U_{\frac{3}{2}}^{N+1}+\left(\frac{1}{k}+\frac{1}{h^{2}}\right) U_{\frac{5}{2}}^{N+1}-\left(\frac{1}{2 h^{2}}\right) U_{\frac{7}{2}}^{N+1} & =\left(\frac{1}{2 h^{2}}\right) U_{\frac{3}{2}}^{N+1}+\left(\frac{1}{k}-\frac{1}{h^{2}}\right) U_{\frac{5}{2}}^{N+1} \\
& +\left(\frac{1}{2 h^{2}}\right) U_{\frac{7}{2}}^{N+1}+\frac{1}{2}\left(F_{\frac{5}{2}}^{N}+F_{\frac{5}{2}}^{N+1}\right)
\end{aligned}
$$

Esta ecuación es una expresión estándar del método de diferencias finitas.

\section{Aproximación en el quinto nodo $x_{\frac{7}{2}}$}

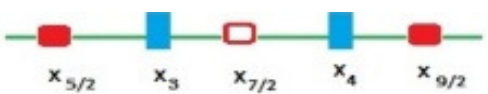

FIGURA 2.7. Aproximación en el cuarto punto medio

Aplicando la ecuación (2.9) en el segundo punto medio, resulta.

$$
\begin{aligned}
\left(-\frac{1}{2 h^{2}}\right) U_{\frac{5}{2}}^{N+1} & +\left(\frac{1}{k}+\frac{1}{12 h}+\frac{1}{h^{2}}\right) U_{\frac{7}{2}}^{N+1}+\left(-\frac{1}{4 h}-\frac{1}{2 h^{2}}\right) U_{\frac{9}{2}}^{N+1}+\left(\frac{1}{6 h}\right) U_{5}^{N+1}=\left(\frac{1}{2 h^{2}}\right) U_{\frac{5}{2}}^{N} \\
& +\left(\frac{1}{k}-\frac{1}{12 h}-\frac{1}{h^{2}}\right) U_{\frac{7}{2}}^{N}+\left(\frac{1}{4 h}+\frac{1}{2 h^{2}}\right) U_{\frac{9}{2}}^{N}-\left(\frac{1}{6 h}\right) U_{5}^{N}+\frac{1}{2}\left(F_{\frac{7}{2}}^{N}+F_{\frac{7}{2}}^{N+1}\right)
\end{aligned}
$$


Donde esta ecuación es simétrica respecto a la aproximación en el tercer nodo ,es decir en $\left(x_{\frac{3}{2}}\right)$.

\section{Aproximación en el sexto nodo $\left(x_{\frac{9}{2}}\right)$}

Verificando la ecuación (2.9) en el quinto punto medio se obtiene:

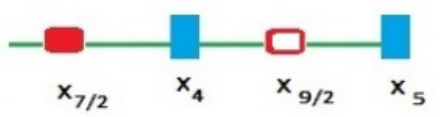

FIGURA 2.8. Aproximación en el quinto punto medio

$$
\begin{aligned}
\left(-\frac{1}{12 h}-\frac{2}{3 h^{2}}\right) U_{\frac{7}{2}}^{N+1} & +\left(\frac{1}{k}+\frac{1}{4 h}+\frac{2}{h^{2}}\right) U_{\frac{9}{2}}^{N+1}+\left(-\frac{1}{6 h}-\frac{4}{3 h^{2}}\right) U_{5}^{N+1}=\left(\frac{1}{12 h}+\frac{2}{3 h^{2}}\right) U_{\frac{7}{2}}^{N} \\
& +\left(\frac{1}{k}-\frac{1}{4 h}-\frac{2}{h^{2}}\right) U_{\frac{9}{2}}^{N}+\left(\frac{1}{6 h}+\frac{4}{3 h^{2}}\right) U_{5}^{N}+\frac{1}{2}\left(F_{\frac{9}{2}}^{N}+F_{\frac{9}{2}}^{N+1}\right)
\end{aligned}
$$

Además se observa que esta ecuación es simétrica respecto a la aproximación del segundo nodo, es decir en $\left(x_{\frac{1}{2}}\right)$.

Aproximación en el último nodo $\left(x_{5}\right)$

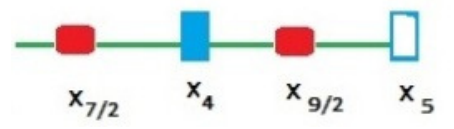

FIGURA 2.9. Aproximación en la frontera derecha

Aplicando la ecuación (2.9) en la frontera derecha se obtiene:

$$
\begin{aligned}
\left(\frac{1}{6 h}\right) U_{\frac{7}{2}}^{N+1}-\left(\frac{3}{2 h}\right) U_{\frac{9}{2}}^{N+1}+\left(\frac{1}{2} \alpha_{2}+\frac{4}{3 h}\right) U_{5}^{N+1} & =\left(-\frac{1}{6 h}\right) U_{\frac{7}{2}}^{N}+\left(-\frac{3}{2 h}\right) U_{\frac{9}{2}}^{N} \\
& +\left(-\frac{1}{2} \alpha_{2}-\frac{4}{3 h}\right) U_{5}^{N}+\frac{1}{2}\left(f_{5}^{N}+f_{5}^{N+1}\right)
\end{aligned}
$$

La cual es simétrica respecto a la aproximación del primer nodo, es decir en $\left(x_{0}\right)$.

Aplicando la ecuación (1.14)

$$
M I=\left(\begin{array}{ccccccc}
\alpha_{1}+\frac{8}{3 h} & -\frac{3}{h} & \frac{1}{3 h} & 0 & 0 & 0 & 0 \\
-\frac{1}{3 h}-\frac{8}{3 h^{2}} & \frac{1}{2 h}+\frac{4}{h^{2}} & -\frac{1}{6 h}-\frac{4}{3 h^{2}} & 0 & 0 & 0 & 0 \\
\frac{1}{3} & -\frac{1}{2 h}-\frac{1}{h^{2}} & \frac{1}{6 h}+\frac{2}{h^{2}} & -\frac{1}{h^{2}} & 0 & 0 & 0 \\
0 & 0 & -\frac{1}{h^{2}} & \frac{2}{h^{2}} & -\frac{1}{h^{2}} & 0 & 0 \\
0 & 0 & 0 & -\frac{1}{h^{2}} & \frac{1}{6 h}+\frac{2}{h^{2}} & -\frac{1}{2 h}-\frac{1}{h^{2}} & \frac{1}{3 h} \\
0 & 0 & 0 & 0 & -\frac{1}{6 h}-\frac{4}{3 h^{2}} & \frac{1}{2 h}+\frac{4^{2}}{h^{2}} & -\frac{1}{3 h}-\frac{8}{3 h^{2}} \\
0 & 0 & 0 & 0 & \frac{1}{3 h} & -\frac{3}{h} & \alpha_{2}+\frac{8}{3 h}
\end{array}\right)
$$

Se construye la matriz $(T)$, generada por las aproximaciones de los nodos internos, la cual tiene la siguiente forma

$$
T=\left(\begin{array}{ccccccc}
0 & 0 & 0 & 0 & 0 & 0 & 0 \\
0 & \frac{1}{k} & 0 & 0 & 0 & 0 & 0 \\
0 & 0 & \frac{1}{k} & 0 & 0 & 0 & 0 \\
0 & 0 & 0 & \frac{1}{k} & 0 & 0 & 0 \\
0 & 0 & 0 & 0 & \frac{1}{k} & 0 & 0 \\
0 & 0 & 0 & 0 & 0 & \frac{1}{k} & 0 \\
0 & 0 & 0 & 0 & 0 & 0 & 0
\end{array}\right)
$$


donde

$$
\begin{aligned}
T[1,1] & =[0,0], \\
T[7,7] & =[0,0] .
\end{aligned}
$$

Los vectores tanto para el tiempo anterior y posterior:

$$
U=\left(\begin{array}{c}
U_{0}^{N} \\
U_{\frac{1}{2}}^{N} \\
U_{\frac{3}{2}}^{N} \\
U_{\frac{5}{2}}^{N} \\
U_{\frac{7}{2}}^{N} \\
U_{\frac{9}{2}}^{N} \\
U_{5}^{N}
\end{array}\right) \quad V=\left(\begin{array}{c}
U_{0}^{N+1} \\
U_{\frac{1}{2}}^{N+1} \\
U_{\frac{3}{2}}^{N+1} \\
U_{\frac{5}{2}}^{N}+1 \\
U_{\frac{7}{2}}^{N+1} \\
U_{\frac{9}{2}}^{N+1} \\
U_{5}^{N+1}
\end{array}\right)
$$

3. Convergencia de la solución aproximada. La propiedad mas importante de todo esquema en diferencias finitas es la convergencia del método hacia la solución exacta, usaremos el teorema de Lax Friedrichs para probar la convergencia del esquema mimético tipo Crank Nicolson.

De la aproximación en el primer nodo, obtenemos:

$U_{0}^{N+1}=\left(\frac{3}{2 h}\right)\left(\frac{6 h}{3 h \alpha_{1}+8}\right) U_{\frac{1}{2}}^{N+1}-\left(\frac{1}{6 h}\right)\left(\frac{6 h}{3 h \alpha_{1}+8}\right) U_{\frac{3}{2}}^{N+1}-\left(\frac{1}{2} \alpha_{1}+\frac{4}{3 h}\right)\left(\frac{6 h}{3 h \alpha_{1}+8}\right) U_{0}^{N}+$

$$
\left(\frac{3}{2 h}\right)\left(\frac{6 h}{3 h \alpha_{1}+8}\right) U_{\frac{1}{2}}^{N}-\left(\frac{1}{6 h}\right)\left(\frac{6 h}{3 h \alpha_{1}+8}\right) U_{\frac{3}{2}}^{N}+\frac{1}{2}\left(f_{0}^{N+1}+f_{0}^{N}\right)\left(\frac{6 h}{3 h \alpha_{1}+8}\right)
$$

donde obtenemos:

$$
\begin{aligned}
U_{0}^{N+1} & =\left(\frac{9}{3 h \alpha_{1}+8}\right) U_{\frac{1}{2}}^{N+1}-\left(\frac{1}{3 h \alpha_{1}+8}\right) U_{\frac{3}{2}}^{N+1}-U_{0}^{N}+\left(\frac{9}{3 h \alpha_{1}+8}\right) U_{\frac{1}{2}}^{N} \\
& -\left(\frac{1}{3 h \alpha_{1}+8}\right) U_{\frac{3}{2}}^{N}+\left(\frac{6}{3 h \alpha_{1}+8}\right) \gamma_{0}
\end{aligned}
$$

De (3.2) y remplazando en la ecuación generada por la aproximación en el primer punto interno.

$$
\begin{array}{r}
-\frac{h+8}{6 h^{2}}\left[\frac{9}{3 h \alpha_{1}+8} U_{\frac{1}{2}}^{N+1}-\frac{U_{\frac{3}{2}}^{N+1}}{3 h \alpha_{1}+8}-U_{0}^{N}+\frac{9}{3 h \alpha_{1}+8} U_{\frac{1}{2}}^{N}-\frac{U_{\frac{3}{2}}^{N}}{3 h \alpha_{1}+8}+\frac{6 h}{3 h \alpha_{1}+8} \gamma_{0}\right] \\
+\left(\frac{1}{4 h}+\frac{2}{h^{2}}+\frac{1}{k}\right) U_{\frac{1}{2}}^{N+1}+\left(-\frac{1}{12 h}-\frac{2}{3 h^{2}}\right) U_{\frac{3}{2}}^{N+1}+\left(-\frac{1}{6}-\frac{4}{3 h^{2}}\right) U_{0}^{N}-\left(-\frac{1}{4}-\frac{2}{h^{2}}+\frac{1}{k}\right) U_{\frac{1}{2}}^{N} \\
-\left(\frac{1}{12 h}+\frac{2}{3 h^{2}}\right) U_{\frac{3}{2}}^{N}-\frac{1}{2}\left(F_{\frac{1}{2}}^{N+1}+F_{\frac{1}{2}}^{N}\right)=0
\end{array}
$$

donde

$$
\gamma_{0}=\frac{1}{2}\left(f_{0}^{N+1}+f_{0}^{N}\right)
$$

$$
\begin{aligned}
& -\left(\frac{3(h+8)}{\left(2 h^{2}\right)\left(3 h \alpha_{1}+8\right)}\right) U_{\frac{1}{2}}^{N+1}+\left(\frac{h+8}{6 h^{2}\left(3 h \alpha_{1}+8\right)}\right) U_{\frac{3}{2}}^{N+1}+\left(\frac{h+8}{6 h^{2}}\right) U_{0}^{N}-\left(\frac{3(h+8)}{\left(2 h^{2}\right)\left(3 h \alpha_{1}+8\right)}\right) U_{\frac{1}{2}}^{N} \\
& \quad+\left(\frac{h+8}{6 h\left(3 h \alpha_{1}+8\right)}\right) U_{\frac{3}{2}}^{N}-\left(\frac{h+8}{h\left(3 h \alpha_{1}+8\right)}\right) \gamma_{0}+\left(\frac{1}{4 h}+\frac{2}{h^{2}}+\frac{1}{k}\right) U_{\frac{1}{2}}^{N+1}-\left(\frac{1}{12 h}+\frac{2}{3 h^{2}}\right) U_{\frac{3}{2}}^{N+1} \\
& (3.4) \quad+\left(\frac{1}{6 h^{2}}-\frac{4}{3 h^{2}}\right) U_{0}^{N}-\left(-\frac{1}{4 h}-\frac{2}{h^{2}}+\frac{1}{k}\right) U_{\frac{1}{2}}^{N}-\left(\frac{1}{12 h}+\frac{2}{3 h^{2}}\right) U_{\frac{3}{2}}^{N}-\frac{1}{2}\left(F_{\frac{1}{2}}^{N+1}+F_{\frac{1}{2}}^{N}\right)=0
\end{aligned}
$$


Luego

$$
\begin{array}{r}
{\left[-\frac{3}{2 h^{2}}\left(\frac{h+8}{3 h \alpha_{1}+8}\right)+\left(\frac{h+8}{4 h^{2}}\right)+\frac{1}{k}\right] U_{\frac{1}{2}}^{N+1}+\left[\left(\frac{h+8}{6 h^{2}}\right)\left(\frac{1}{3 h \alpha_{1}+8}\right)-\left(\frac{h+8}{12 h^{2}}\right)\right] U_{\frac{3}{2}}^{N+1}} \\
+\left[-\frac{3}{2 h^{2}}\left(\frac{h+8}{3 h \alpha_{1}+8}\right)+\left(\frac{h+8}{4 h^{2}}\right)-\frac{1}{k}\right] U_{\frac{1}{2}}^{N}+\left[\frac{1}{6 h^{2}}\left(\frac{h+8}{3 h \alpha_{1}+8}\right)-\left(\frac{h+8}{12 h^{2}}\right)\right] U_{\frac{3}{2}}^{N}- \\
\left(\frac{h+8}{h\left(3 h \alpha_{1}+8\right)}\right) \gamma_{0}=\frac{1}{2}\left(F_{\frac{1}{2}}^{N+1}+F_{\frac{1}{2}}^{N}\right)
\end{array}
$$

Reemplazando (3.2) en la ecuación generada por la aproximación en el segundo punto medio.

$$
\begin{aligned}
\frac{1}{6 h} & {\left[\frac{9}{3 h \alpha_{1}+8} U_{\frac{1}{2}}^{N+1}-\frac{1}{3 h \alpha_{1}+8} U_{\frac{3}{2}}^{N+1}-U_{0}^{N}+\frac{9}{3 h \alpha_{1}+8} U_{\frac{1}{2}}^{N}-\frac{U_{\frac{3}{2}}^{N}}{3 h \alpha_{1}+8}+\frac{6 h}{3 h \alpha_{1}+8} \gamma_{0}\right] } \\
+ & \left(-\frac{1}{4 h}-\frac{1}{2 h^{2}}\right) U_{\frac{1}{2}}^{N+1}+\left(\frac{1}{12 h}+\frac{1}{h^{2}}+\frac{1}{k}\right) U_{\frac{3}{2}}^{N+1}-\frac{1}{2 h^{2}} U_{\frac{5}{2}}^{N+1}+\frac{1}{6 h} U_{0}^{N}+\left(\frac{1}{4 h}-\frac{1}{2 h^{2}}\right) U_{\frac{1}{2}}^{N}- \\
& \left(\frac{1}{k}-\frac{1}{12 h}-\frac{1}{h^{2}}\right) U_{\frac{3}{2}}^{N}-\left(\frac{1}{2 h^{2}}\right) U_{\frac{5}{2}}^{N}-\frac{1}{2}\left(F_{\frac{3}{2}}^{N+1}+F_{\frac{3}{2}}^{N}\right) \\
& \left(\frac{3}{2 h\left(3 h \alpha_{1}+8\right)}\right) U_{\frac{1}{2}}^{N+1}-\frac{U_{\frac{3}{2}}^{N+1}}{6 h\left(3 h \alpha_{1}+8\right)}-\frac{U_{0}^{N}}{6 h}+\frac{3 U_{\frac{1}{2}}^{N}}{2 h\left(3 h \alpha_{1}+8\right)}-\frac{U_{\frac{3}{2}}^{N}}{6 h\left(3 h \alpha_{1}+8\right)} \\
+ & \left(\frac{1}{3 h \alpha_{1}+8}\right) \gamma_{0}+\left(-\frac{1}{4 h}-\frac{1}{2 h^{2}}\right) U_{\frac{1}{2}}^{N+1}+\left(\frac{1}{12 h}+\frac{1}{h^{2}}+\frac{1}{k}\right) U_{\frac{3}{2}}^{N+1}-\frac{1}{2 h^{2}} U_{\frac{5}{2}}^{N+1}+\frac{1}{6 h} U_{0}^{N}- \\
& \left(-\frac{1}{4 h}+\frac{1}{2 h^{2}}\right) U_{\frac{1}{2}}^{N}-\left(\frac{1}{k}-\frac{1}{12 h}-\frac{1}{h^{2}}\right) U_{\frac{3}{2}}^{N}-\left(\frac{1}{2 h^{2}}\right) U_{\frac{5}{2}}^{N}-\frac{1}{2}\left(F_{\frac{3}{2}}^{N+1}+F_{\frac{3}{2}}^{N}\right)
\end{aligned}
$$

Finalmente se obtiene:

$$
\begin{aligned}
& {\left[\frac{3}{2 h\left(3 h \alpha_{1}+8\right)}-\frac{h+2}{4 h^{2}}\right] U_{\frac{1}{2}}^{N+1}+\left[-\frac{1}{6 h\left(3 h \alpha_{1}+8\right)}+\frac{h+12}{12 h^{2}}+\frac{1}{k}\right] U_{\frac{3}{2}}^{N+1}} \\
& -\frac{1}{2 h^{2}} U_{\frac{5}{2}}^{N+1}+\left[\frac{3}{2 h\left(3 h \alpha_{1}+8\right)}-\frac{h+2}{4 h^{2}}\right] U_{\frac{1}{2}}^{N}+\left[-\frac{1}{6 h\left(3 h \alpha_{1}+8\right)}+\frac{h+12}{12 h^{2}}-\frac{1}{k}\right] U_{\frac{3}{2}}^{N} \\
& -\frac{1}{2 h^{2}} U_{\frac{5}{2}}^{N}+\frac{\gamma_{0}}{3 h \alpha_{1}+8}=\frac{1}{2}\left(F_{\frac{3}{2}}^{N+1}+F_{\frac{3}{2}}^{N}\right)
\end{aligned}
$$

Como $h$ es el tamaño de paso en la variable espacial entonces la expresión:

$$
\left(\frac{h+8}{h\left(3 h \alpha_{1}+8\right)}\right)
$$

es diferente de cero, independiente al valor que puede asumir la constante $\alpha_{1}$.Luego se tiene:

$$
\begin{aligned}
& \left(\frac{h+8}{h\left(3 h \alpha_{1}+8\right)}\right)\left[\left(\frac{\alpha_{1}}{2}+\frac{4}{3 h}\right) U_{0}^{N+1}-\frac{3}{2 h} U_{\frac{1}{2}}^{N+1}+\frac{1}{6 h} U_{\frac{3}{2}}^{N+1}\right] \\
& \quad+\left(\frac{h+8}{h\left(3 h \alpha_{1}+8\right)}\right)\left[\left(\frac{\alpha_{1}}{2}+\frac{4}{3 h}\right) U_{0}^{N}-\frac{3}{2} U_{\frac{1}{2}}^{N}+\left(\frac{1}{6 h}\right) U_{\frac{3}{2}}^{N}-\gamma_{0}\right]=0
\end{aligned}
$$


De la ecuación dada en (3.7) y la aproximación del primer punto medio tenemos:

$$
\begin{aligned}
\left(-\frac{1}{6 h}-\frac{4}{3 h^{2}}\right) U_{0}^{N+1} & +\left(\frac{1}{4 h}+\frac{2}{h^{2}}+\frac{1}{k}\right) U_{\frac{1}{2}}^{N+1}-\left(\frac{1}{12 h}+\frac{2}{3 h^{2}}\right) U_{\frac{3}{2}}^{N+1}-\left(\frac{1}{6 h}+\frac{4}{3 h^{2}}\right) U_{0}^{N} \\
& -\left(-\frac{1}{4 h}-\frac{2}{h^{2}}+\frac{1}{k}\right) U_{\frac{1}{2}}^{N}-\left(-\frac{1}{12 h}+\frac{2}{3 h^{2}}\right) U_{\frac{3}{2}}^{N}-\frac{1}{2}\left(F_{\frac{1}{2}}^{N+1}+F_{\frac{1}{2}}^{N}\right) \\
& +\frac{h+8}{h\left(3 h \alpha_{1}+8\right)}\left[\left(\frac{\alpha_{1}}{2}+\frac{4}{3 h}\right) U_{0}^{N+1}-\left(\frac{3}{2 h}\right) U_{\frac{1}{2}}^{N+1}+\left(\frac{1}{6 h}\right) U_{\frac{3}{2}}^{N+1}\right] \\
& +\frac{h+8}{h\left(3 h \alpha_{1}+8\right)}\left[\left(\frac{\alpha_{1}}{2}+\frac{4}{3 h}\right) U_{0}^{N}-\left(\frac{3}{2 h}\right) U_{\frac{1}{2}}^{N}+\left(\frac{1}{6 h}\right) U_{\frac{3}{2}}^{N}\right]-\gamma_{0}=0
\end{aligned}
$$

La expresión dada por

$$
\left(\frac{1}{h\left(3 h \alpha_{1}+8\right)}\right)
$$

es una cantidad diferente de cero. Luego:

$$
\begin{array}{r}
-\left(\frac{1}{3 h \alpha_{1}+8}\right)\left[\left(\frac{\alpha_{1}}{2}+\frac{4}{3 h}\right) U_{0}^{N}-\frac{3}{2 h} U_{\frac{1}{2}}^{N}+\frac{1}{6 h} U_{\frac{3}{2}}^{N}-\gamma_{0}\right]-\left(\frac{1}{3 h \alpha_{1}+8}\right) \\
{\left[\left(\frac{\alpha_{1}}{2}+\frac{4}{3 h}\right) U_{0}^{N+1}-\frac{3}{2 h} U_{\frac{1}{2}}^{N+1}+\frac{1}{6 h} U_{\frac{3}{2}}^{N+1}\right]=0}
\end{array}
$$

Considerando la ecuación (3.9) y la aproximación en el segundo punto medio, se tiene:

$$
\begin{aligned}
\frac{1}{6 h}\left(U_{0}^{N+1}+U_{0}^{N}\right) & +\left(-\frac{1}{4 h}-\frac{1}{2 h^{2}}\right) U_{\frac{1}{2}}^{N+1}+\left(\frac{1}{k}+\frac{1}{12 h}+\frac{1}{h^{2}}\right) U_{\frac{3}{2}}^{N+1}-\left(\frac{1}{2 h^{2}}\right) U_{\frac{5}{2}}^{N+1} \\
& -\left(\frac{1}{4 h}+\frac{1}{2 h^{2}}\right) U_{\frac{1}{2}}^{N}-\left(\frac{1}{k}-\frac{1}{12 h}-\frac{1}{h^{2}}\right) U_{\frac{3}{2}}^{N}-\frac{1}{2 h^{2}} U_{\frac{5}{2}}^{N}-\frac{1}{2}\left(F_{\frac{3}{2}}^{N}+F_{\frac{3}{2}}^{N+1}\right) \\
& -\left(\frac{1}{3 h \alpha_{1}+8}\right)\left[\left(\frac{\alpha_{1}}{2}+\frac{4}{3 h}\right) U_{0}^{N}-\frac{3}{2 h} U_{\frac{1}{2}}^{N}+\frac{1}{6 h} U_{\frac{3}{2}}^{N}-\gamma_{0}\right] \\
& -\left(\frac{1}{3 h \alpha_{1}+8}\right)\left[\left(\frac{\alpha_{1}}{2}+\frac{4}{3 h}\right) U_{0}^{N+1}-\frac{3}{2 h} U_{\frac{1}{2}}^{N+1}+\frac{1}{6 h} U_{\frac{3}{2}}^{N+1}\right]=0
\end{aligned}
$$

Se asume que $k=\Delta t$.

Observamos que las ecuaciones dadas en (3.6) y (3.7) podemos escribirlas como(3.8) y (3.9).

Estas expresiones contienen las aproximaciones de la ecuación diferencial y las condiciones de contorno en una sola ecuación.

Por una expansión de Taylor se tiene:

Para la ecuación centrada en el nodo $x_{\frac{1}{2}}$

$$
\frac{\partial U}{\partial t}-\frac{\partial^{2} U}{\partial x^{2}}-\frac{1}{6} h \frac{\partial U^{3}}{\partial x^{3}}+\frac{1}{24} \Delta t^{2} \frac{\partial U^{3}}{\partial t^{3}}
$$

el error de truncamiento es $O(h)+O\left(\Delta t^{2}\right)$.

Para la ecuación centrada en el nodo $x_{\frac{3}{2}}$

$$
\frac{\partial U}{\partial t}-\frac{\partial^{2} U}{\partial x^{2}}-\frac{5}{48} h^{2} \frac{\partial U^{3}}{\partial x^{3}}+\frac{1}{24} \Delta t^{2} \frac{\partial U^{3}}{\partial t^{3}}
$$

el error de truncamiento es $O\left(h^{2}\right)+O\left(\Delta t^{2}\right)$.

Para la ecuación centrada en el nodo $x_{i+\frac{1}{2}}$

$$
\frac{\partial U}{\partial t}-\frac{\partial^{2} U}{\partial x^{2}}-\frac{1}{12} h^{2} \frac{\partial U^{4}}{\partial x^{4}}-\frac{1}{8} \Delta t^{2} \frac{\partial U^{4}}{\partial t^{4}}
$$


el error de truncamiento es $O\left(h^{2}\right)+O\left(\Delta t^{2}\right)$.

Los errores de truncamiento para las ecuaciones centradas en los nodos $x_{N-\frac{3}{2}}$ y $x_{N-\frac{1}{2}}$ son iguales a las ecuaciones (3.12) y (3.11).

Luego se escribe el sistema asociado usando las ecuaciones de aproximación en el cuarto nodo,(3.8) y (3.10):

$$
(I+r W) U^{N+1}=(I-r W) U^{N}
$$

donde $r=\frac{\Delta t}{h^{2}}$ y sus valores propios son $\mu=\frac{(1-r \lambda)}{(1+r \lambda)}$.

Por último se demuestra que $\mu \leq 1$. Para obtener este resultado, debemos verificar que $\lambda \geq 0$. Esto se hace aplicando el teorema de Gershgorin en cada ecuación del sistema (3.14), por lo que el radio espectral es menor o igual de uno y significa que el nuevo método es incondicionalmente estable y por el teorema de Lax-Friedrichs es convegente. Más detalles de esta prueba en [10] y [1].

\section{Resultados.}

4.1. Estudio numérico comparativo. Se presenta un problema de prueba unidimensional resuelto con el nuevo esquema mimético - Crank Nicolson comparándolo con el esquema de diferencia finita tipo Crank Nicolson.

$$
\frac{\partial u}{\partial t}-\frac{\partial^{2} u}{\partial x^{2}}=-\frac{1}{10} e^{-\frac{t}{10}} \operatorname{sen}(2 \pi x)+4 e^{-\frac{t}{10}} \operatorname{sen}(2 \pi x) \pi^{2}
$$

definido en $(0,1)$ y las condiciones de frontera de Robín

$$
\begin{aligned}
& u(0)-\frac{\partial u}{\partial x}(0)=-2 \pi e^{\frac{-t}{10}} \\
& u(1)+\frac{\partial u}{\partial x}(1)=2 \pi e^{\frac{-t}{10}}
\end{aligned}
$$

con condición inicial

$$
u(x, 0)=\operatorname{sen}(2 \pi x)
$$

Las ecuaciones (4.1), (4.2) y (4.3) muestran un problema matemático bien planteado con solución analítica:

$$
u(x, t)=e^{\frac{t}{10}} \operatorname{sen}(2 \pi x)
$$


4.2. Algoritmo en Matlab. A continuación se detalla el algoritmo en Matlab para determinar el error entre la solución exacta y la aproximada.

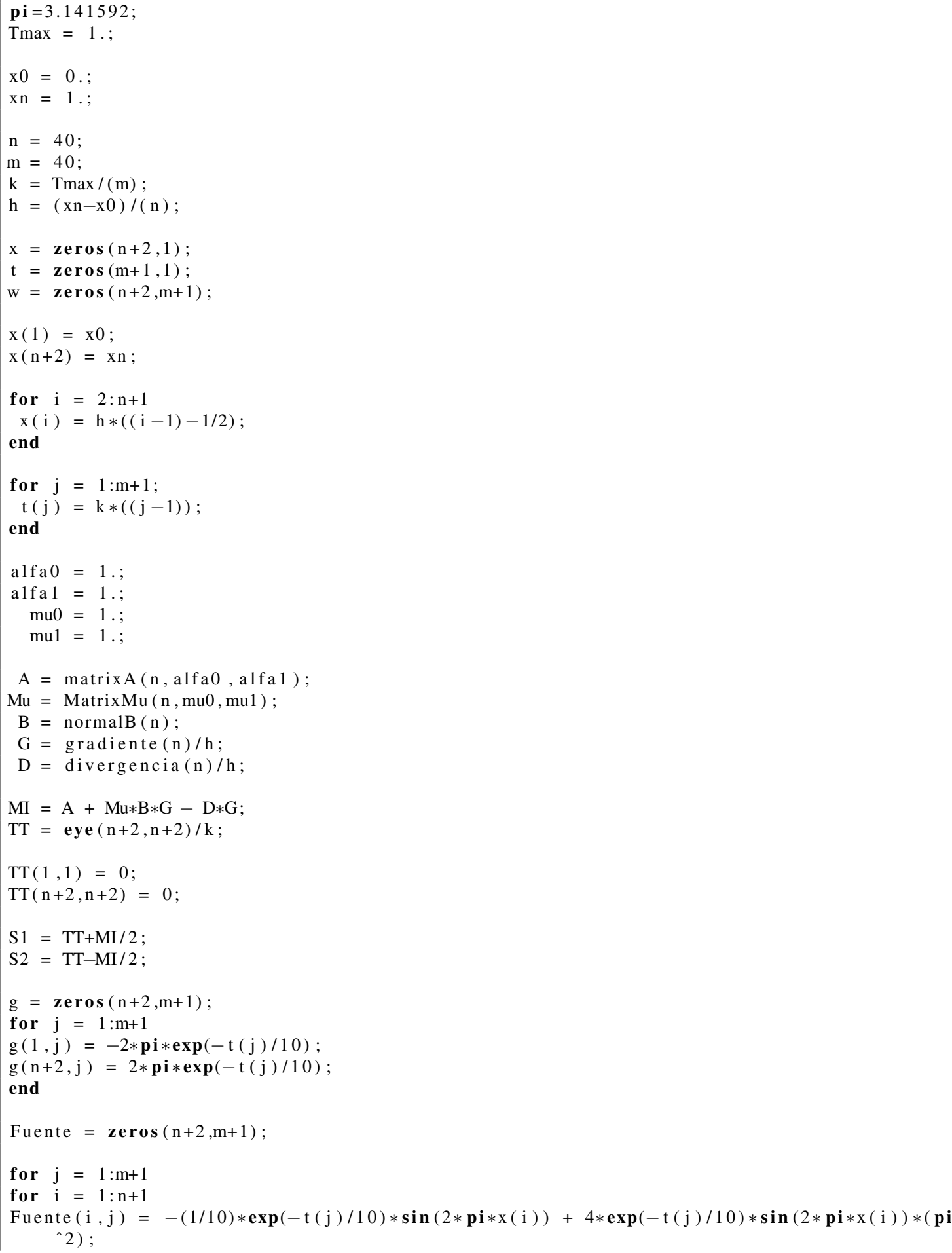




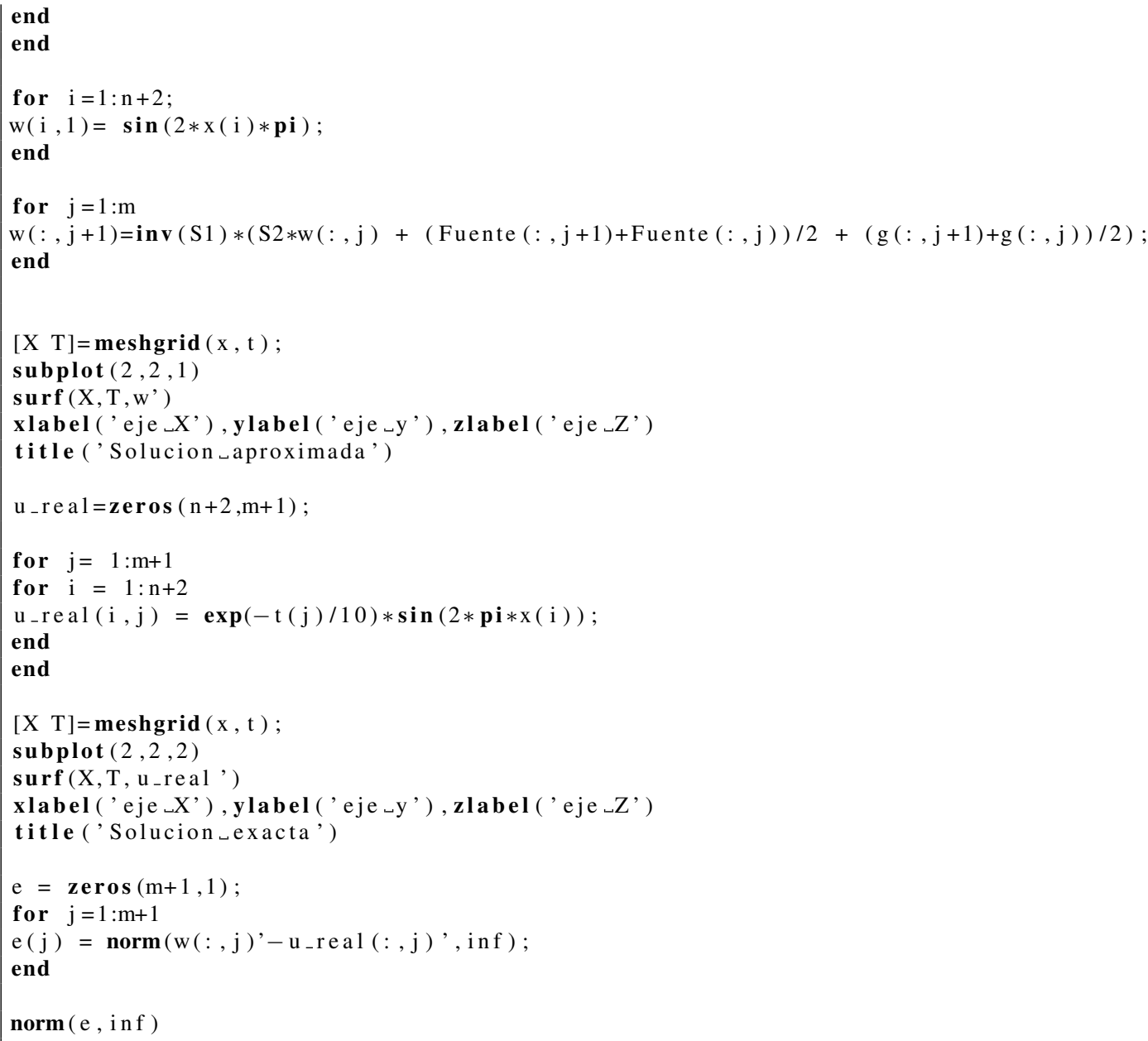

El error numérico computacional entre la solución exacta y la aproximada usando la norma del máximo y bajo el enfoque en diferencias finitas miméticas generan los siguientes resultados.

CUADRO 4.2

Variación del error de aproximación para distinto tamaños de malla.

\begin{tabular}{|c|c|c|}
\hline \hline Método & Tamaño & Error \\
\hline & 40 & 0.0031 \\
Mimético & 70 & 0.0010 \\
& 100 & $4.9832 e^{-04}$ \\
\hline \multirow{2}{*}{ Diferencias } & 40 & 0.0049 \\
Finitas & 70 & 0.0015 \\
& 100 & $7.1630 e^{-04}$ \\
\hline
\end{tabular}

El cuadro (4.2) muestra la longitud de los errores relativos producidos por métodos miméticos y de diferencias finitas. Los primeros son más pequeños que los segundos.

El error de aproximación es el más óptimo cuando hacemos más densa la partición de la malla, para una partición de malla de $40 \times 40$ obtenemos un error de 0,0031 , luego si tomamos una partición en la malla de $70 \times 70$ se obtiene un resultado de 0,0010, esto demuestra que el error de aproximación entre la solución exacta y la discreta mejora cada vez que generamos más puntos nodales en el mallado.

Del algoritmo descrito anteriormente, se construyen las gráficas tanto para la solución exacta y la solución aproximada de la ecuación de difusión no estacionaria en un medio unidimensional con condiciones de frontera tipo Robín, en un mallado escalonado y uniforme del dominio. 


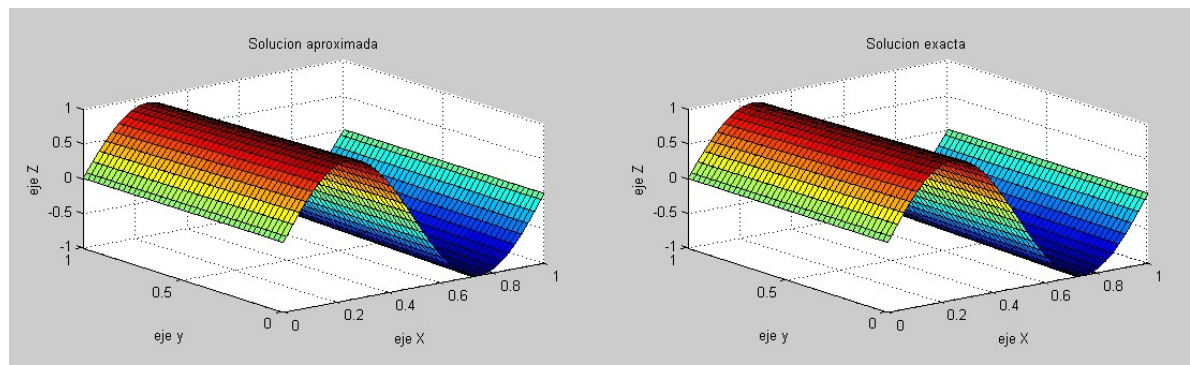

FIgURA 4.1. Gráfica usando esquema Mmético para una malla $n=m=40$

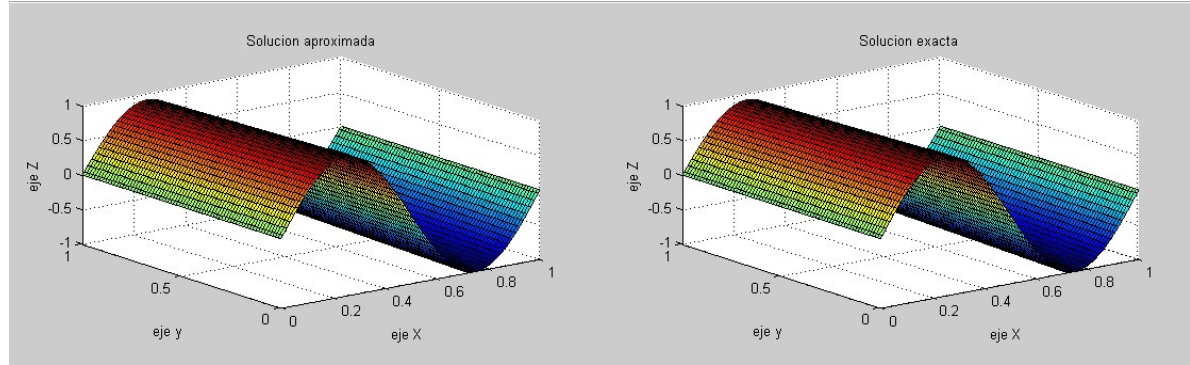

FIGURA 4.2. Gráfica usando esquema Mmético para una malla $n=m=70$

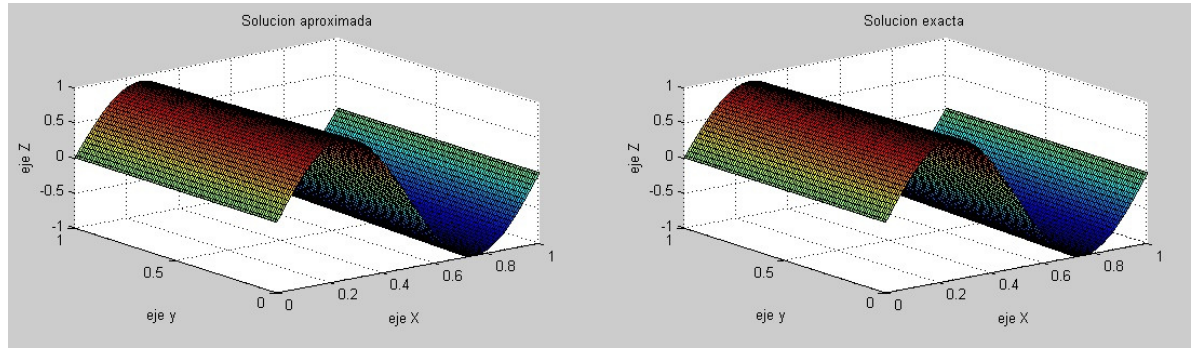

FIGURA 4.3. Gráfica usando esquema Mmético para una malla $n=m=100$

El análisis hecho en las gráficas (4.1), (4.2) y (4.3) en la construcción geométrica de la solución exacta para la ecuación de difusión no estacionaria unidimensional ha llevado a establecer la relación entre la solución real sobre los puntos de la malla, permitiéndonos comparar su respectiva solución numérica, esta comparación muestra que la solución discreta presenta una ligera variación respecto a la gráfica de la solución exacta en una malla regular de puntos nodales distribuidos de forma homogénea.

\section{Conclusiones.}

En el presente trabajo se desarrolló un algoritmo que combina el esquema de diferencias finitas miméticas y el esquema de Crank Nicolson para determinar la solución aproximada hacia la solución exacta de la ecuación de difusión en estado no estacionario en un medio unidimensional con condiciones de frontera mixtas.

Se corrió el programa con el siguiente informe detallado en las figuras (4.1) y (4.2), el cual muestra un error de aproximación óptimo respecto a la partición del mallado unidimensional, es así que el tratamiento de las condiciones de contorno no implica el uso de mallas extendidas ni la inclusión de puntos fantasmas en la formulación del esquema en diferencias finitas miméticas, como ocurre a veces con el método estándar de diferencias finitas tipo Crank Nicolson, la cual hace su implementación numérica más simple.

Los operadores discretos provenientes del método mimético de Castillo-Grone se caracterizan por mantener los mismos órdenes de exactitud tanto en el interior como en la frontera del dominio. 


\section{Referencias}

[1] Mannarino, I.A. A mimetic finite difference method using Crank Nicolson scheme for unsteady diffusion equation. Revista de matemática: Teoría y Aplicaciones. 2009; 16(2): 221 - 230.

[2] Castillo, J.E. and Grone, R.D. A matrix analysis approach to higher-order aproximations for divergence and gradients satisfying a global conservation law. SIAM Journal on Matrix Analysis and Applications. 2003; 25(1): 128 - 142.

[3] Castillo, J.E. and Yasuda, M. Linear system arising for second order mimetic divergence and gradient operators. Journal of Mathematical Modeling and Algorithm. 2005; 4(1): 67-82.

[4] Guevara- Jordan, J.M.; Rojas, S.; Freites-Villegas, M. and Castillo, J.E. Convergence of a mimetic finite difference method for static diffusion equation. Advances in Difference Equations. 2007; (2007).

[5] Castillo J. and Miranda, G. Mimetic discretization methods. 2013

[6] Mannarino, I.; Quintana, Y. and Guevara-Jordan, J.M A numerical study of mimetic scheme for the unsteady heat equation. submitted fo FACYT Review.

[7] Sánchez, E. Finite Differences and Parallel Computing to Simulate Carbon Dioxide Subsurface Mass Transport. 2015.

[8] Sanchez, E.; Paolini, C. and Castillo, J.E. Analyzing Diffusive-Advective-Reactive Processes Using Mimetic Finite Differences with implications in Carbon Dioxide Geologic Storange. 2014

[9] Gyrya, V.; Koustantin, L. and Manzini, G. The arbitrary order mixed mimetic finite difference method for the diffusion equation. 2016 ; 50(3), 851-877.

[10] Mannarino, I. Un método mimético de diferencias finitas para la ecuación no estática de difusión. Master thesis, Universidad Central de Venezuela, Caracas (2007). 